\title{
Collaborative Filtering Based on Opportunistic Information Sharing in Mobile Ad-Hoc Networks
}

\author{
Alexandre de Spindler, Moira C. Norrie, and Michael Grossniklaus \\ Institute for Information Systems, ETH Zurich \\ CH-8092 Zurich, Switzerland \\ \{despindler, norrie, grossniklaus\}@inf.ethz.ch
}

\begin{abstract}
Personal mobile devices and mobile ad-hoc networks can support interesting forms of opportunistic information sharing in user communities based on spatio-temporal proximity. We show how this could be used to realise a novel decentralised collaborative filtering (CF) approach in a mobile environment.
\end{abstract}

\section{Introduction}

The issue of information sharing in mobile ad-hoc networks is often seen as the problem of how to ensure that users can access remote data in networks without a fixed topology and with possible disconnections. However, the ad-hoc nature of establishing network connections between personal mobile devices can be viewed as a means of sharing information opportunistically among members of a user community based on spatio-temporal proximity.

Although projects such as AIDE [1] and TRACE [2] have investigated the use of physical copresence as a means of forming social networks and opportunistic sharing of information, they have not considered how collaborative filtering algorithms could be adapted to base user similarity on shared social contexts. Our goal was to do exactly that and investigate the use of peer-to-peer architectures to allow users to exchange data automatically and unobtrusively based on spatio-temporal proximity.

We motivate our approach in Sect. 2 and then present our collabortaive filtering algorithm in Sect. 3. In Sect. 4, we show how our approach is equivalent to existing collaborative filtering techniques based on centralised servers. Concluding remarks are given in Sect. 5 .

\section{Motivation}

Recommender systems based on collaborative filtering (CF) have become wellknown through their use in on-line stores. The underlying assumption is that users who bought the same items in the past are likely to do so in the future. One of the first approaches developed was user-based CF [3], in which the opinions of a set of users judged to be similar to the current one are aggregated. The similarity 
between users is measured in terms of the extent to which their opinions about other items correlate. User-based CF has been deployed in a wide variety of application domains such as music, video and web page recommendations $[4,5,6]$. There are three main shortcomings of user-based CF. Firstly, the set of opinions given by a user is usually sparse and so the number of commonly rated items will be small leading to an inaccurate similarity measure. Secondly, the complexity of selecting a set of similar users grows with the number of users and items as $O(\mid$ users $|\times|$ items $\mid)$ leading to problems of scalability. Thirdly, when new users or items are introduced there is a lack of data on which to base recommendations.

A number of approaches address the shortcomings of user-based CF while retaining its advantages. Sarwar et al. [7] introduced the idea of item-based $\mathrm{CF}$ where recommendation is based on the similarity of items rather than users. User- and item-based CF are the best known representatives of so called memorybased approaches which perform filtering based on the raw data. In contrast, model-based approaches compute intermediate representations of the set of the tuples such as clusters, probability distribution functions or singular value decompositions. Model-based approaches effectively resolve the sparsity issue and render predictions more efficient and supposedly accurate.

Most collaborative filtering systems have been designed to be deployed in clientserver architectures whereas only a few approaches $[8,9,10]$ have tackled the challenges of decentralised environments. Distributed filtering research has mainly been concerned with the availability of data on client devices where network connectivity cannot be guaranteed and opinions need to be predicted. Mobile environments introduce additional challenges as limitations of size and power capacity place restricitions on computational power and human computer interface facilities. Despite the advantages of model-based CF in comparison with memory-based approaches, computing the intermediary representation emerges as a new bottleneck, in particular with regard to the limited computational power available on mobile devices. Further, although wireless connectivity is increasingly available within restricted areas such as restaurants and airports as well as public areas by means of $3 \mathrm{G}$ networks, area-wide connectivity is still bound to expensive communication costs, high power consumption and prone to disconnections. In contrast, devices may connect to each other in an ad-hoc peer-to-peer fashion based on short range connectivity technology such as Wi-Fi and Bluetooth. Consequently, a $\mathrm{CF}$ protocol for mobile environments must respect the following requirements. All computation and storage must be decentralised since a connection to a central server may not be available. Due to restricted computational and storage capacities of mobile devices, local computation must be kept simple and the required data small. Ideally, the protocol should rely on ad-hoc peer-to-peer connections only. This transient connectivity requires data exchange to be short and to consume little bandwidth. Additionally, the protocol must be delay tolerant since other peers may not always be available. Finally, since mobile devices typically feature reduced interaction facilities, user interaction should be minimal.

We believe that the notion of shared social contexts can be exploited to establish a similarity relationship between users. For example, if two users attend the 
same music concert, it is likely that they have similar musical tastes. Our initial studies carried out at an international arts festival show that this can be taken further since users who share music preferences often share preferences for other items such as festival events, films and books. As we will show in the following section, this fact can be used to reduce computing costs of $\mathrm{CF}$ as well as to render $\mathrm{CF}$ suitable for ad-hoc connectivity available in mobile environments.

\section{Spatio-temporal Collaborative Filtering}

The application domain of CF contains users consuming items and expressing opinions about these items. Based on these, a collaborative filtering system predicts their opinion about items unknown to them. Opinions are tuples of the form (user, item, value) which can be seen as a directed weighted edge in a graph, pointing from a user node to an item node and weighted with a rating value. Thus, a set of tuples defines a directed graph $G=(U \cup I, E)$ where $U$ is the set of nodes representing users, $I$ the set of item nodes and $E$ the set of directed weighted edges pointing from nodes in $U$ to nodes in $I$.

User-based $\mathrm{CF}$ processes a fundamental query by first computing similarities among users and selecting those judged to be similar. Then the ratings of target items by these users are aggregated. In order to include user similarities, we augment the previously defined graph with undirected edges connecting two users and weighted with their similarities. Thus, the set of edges $E$ is now composed of $E_{r} \cup E_{s}$ where $E_{r}$ contains the rating edges and $E_{s}$ the similarity edges. As proposed by Mirza et al. [11], $G_{s}=\left(U, E_{s}\right)$ represents a social network graph while $G_{r}=\left(U \cup I, E_{r}\right)$ refers to the rating graph.

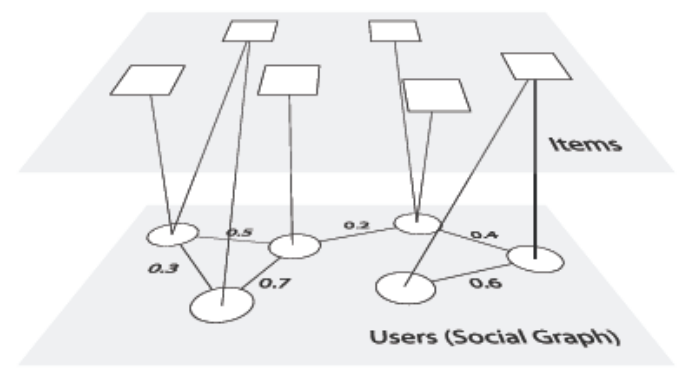

Fig. 1. Social- and rating graph

Figure 1 shows an example graph composed of a social and rating graph. The vertices on the bottom layer represent users and the ones on the top layer items. Edges connecting users are weighted with the similarity of the adjacent users. For clarity, we omit the weights of the edges connecting a user to an item representing the rating value.

In our approach, the selection of users is performed implicitly and without any prior similarity computations. We introduce the concept of spatio-temporal 
proximity which forms the basis for our selection of similar users. In the case of social contexts formed around consumable items, user consumption of an item means that their location matches the location of the item for a specific period of time. Some items such as restaurants or bars can be consumed at any time within predefined opening hours and the duration of consumption can be anything from the time to drink a glass of wine up to eating a dinner. In contrast, items such as comedy shows or theatre plays can be consumed only during a specific time period and the duration is usually well defined. We will refer to these two kinds of items as location and event items, respectively. Note that event items may happen only once or be repeated periodically. All items have in common the fact that if users meet while consuming them, they stay in each other's vicinity for longer than if they would pass each other in the street by chance.

The history of item consumption of a particular user $u_{a}$ can be regarded as a set of item consumption tuples of the form $\left(l o c_{i},\left[t_{k}, t_{l}\right]\right)$ where each tuple contains two entries. The first entry identifies a location $l o c_{i}$ particular to an item. This location represents an area in which the item can be consumed. The second one delimits a period of time $\left[t_{k}, t_{l}\right]$ during which the item was consumed. Consequently, the history $H\left(u_{a}\right)$ of a user $u_{a}$ can be written as $H\left(u_{a}\right)=$ $\left\{\left(l_{o c_{1}},\left[t_{1}, t_{2}\right]\right),\left(l o c_{2},\left[t_{3}, t_{4}\right]\right), \ldots\right\}$. The condition for item consumption tuples to be equal is $\left(l o c_{i},\left[t_{k}, t_{l}\right]\right)=\left(\operatorname{loc}_{j},\left[t_{m}, t_{n}\right]\right) \Longleftrightarrow\left(l o c_{i}=l o c_{j}\right) \wedge\left(\left[t_{k}, t_{l}\right] \cap_{t}\left[t_{m}, t_{n}\right] \geq\right.$ $p$ ) where we define $\cap_{t}$ as a temporal intersection of two time periods. The condition $\left[t_{k}, t_{l}\right] \cap_{t}\left[t_{m}, t_{n}\right] \geq p$ holds if the time periods overlap for a duration of at least $p$. The first component $\left(l o c_{i}=l o c_{j}\right)$ accounts for spatial proximity while the temporal intersection accounts for temporal proximity.

The user similarity $P_{l o c, t}$ resulting from spatio-temporal proximity between two users $u_{a}$ and $u_{b}$ can be expressed as

$$
P_{l o c, t}^{\mathbb{N}}\left(u_{a}, u_{b}\right)= \begin{cases}1 & \text { if } H\left(u_{a}\right) \cap H\left(u_{b}\right) \neq \emptyset \\ 0 & \text { else. }\end{cases}
$$

This is a binary similarity measure in the sense that users are evaluated to be similar only if they have at least one tuple of their consumption history in common. We use $P_{l o c, t}^{\mathbb{N}}\left(u_{a}, u_{b}\right)$ as a condition for the users $u_{a} \in U$ and $u_{b} \in U$ to be connected by a similarity edge $\left(u_{a}, u_{b}\right) \in E_{s}$ in the social graph $G_{s}$. The resulting social graph corresponds to a copresence community used by Lawrence et al. [1] to disseminate information since spatio-temporal proximity is a necessary and sufficient condition for users to have their devices connected.

We can refine this similarity taking into consideration the level of spatiotemporal proximity among users. Based on the fact that users consuming the same items are similar, it is obvious that the more often users consume the same item, the more similar they are. This calls for a continuous similarity measure $P_{l o c, t}^{\mathbb{R}}$ that takes into account the number of common simultaneous item consumptions as opposed to the binary measure proposed before.

$$
P_{l o c, t}^{\mathbb{R}}\left(u_{a}, u_{b}\right)= \begin{cases}\frac{\left|H\left(u_{a}\right) \cap H\left(u_{b}\right)\right|}{\max \left(\left|H\left(u_{a}\right)\right|,\left|H\left(u_{b}\right)\right|\right)} & \text { if } H\left(u_{a}\right) \neq \emptyset \\ 0 & \text { else }\end{cases}
$$


This measure allows us to assign a weight to a similarity edge created based on the binary measure. Note that if it evaluates to zero, the respective users are not connected in the graph while it never evaluates to zero if they are connected.

We now describe our CF approach in terms of a formal description of the algorithm running on a single mobile device as shown in Figure 2. For this discussion, we assume the existence of three library functions. WAIT $(p)$ causes the algorithm to pause for a time period of $p$, Transmit $($ Peer,$M)$ transmits a set of edges $M$ to a remote peer Peer. This transmission will be translated to a call of the function $\operatorname{RECEIVE}(M)$ on the remote peer where $M$ corresponds to the second argument of the transmission function. InCREASE-WEIGHT(Peer) retrieves the edge $\left(u_{\text {local }}, u_{\text {remote }}\right) \in E_{s}$ where $u_{\text {remote }}$ denotes the user node representing the argument Peer and increases its weight in order to update the respective continuous proximity value.
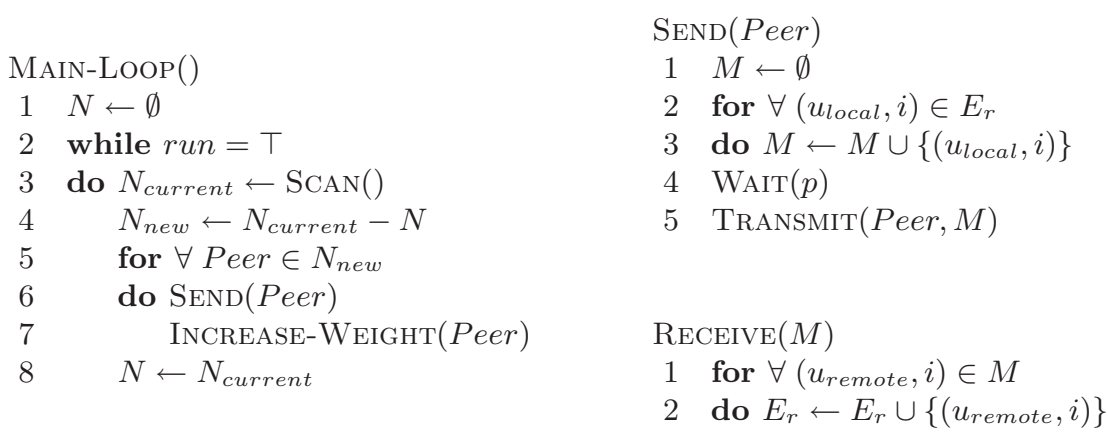

Fig. 2. Collaborative filtering algorithm

While a peer is active, i.e. run $=\top$, the main loop simply scans the environment periodically and maintains a set $N$ of peers in the vicinity. For every remote peer Peer in the vicinity, the method $\operatorname{SEND}(P e e r)$ is called to send all ratings made by the local user to the remote peer. This method runs as a thread per remote peer in order to be non-blocking. Note that these ratings will only be sent after a delay of length $p$, the parameter introduced above to determine the equality of two rating consumption tuples. If the remote peer has left the vicinity of the local peer during this time period, the tuples will not be sent by the Transmit $($ Peer, $M)$ function to avoid exchanges during a transient encounter. Once the rating tuples have been sent to all new peers in the vicinity, the set of peers in the vicinity is updated to remove peers that have left. Whenever a local peer receives a set of tuples from a remote peer, $\operatorname{RECEIVE}(M)$ is called and these tuples are added to the set of tuples stored locally.

Finally, rating values from similar users about the target item are aggregated. The most common approach is to compute the average. To do so, we select all incoming edges of the node representing the target item and compute the average of their weights. We also take into account the degree of similarity as expressed by the continuous proximity measure. $P_{l o c, t}^{\mathbb{R}}\left(u_{a}, u_{b}\right)$ establishes a ranking of the users according to their similarity to the user denoted by the first argument. A 
user $u_{a}$ is more similar to a user $u_{b}$ than to another user $u_{c}$ if $P_{l o c, t}^{\mathbb{R}}\left(u_{a}, u_{b}\right)>$ $P_{l o c, t}^{\mathbb{R}}\left(u_{a}, u_{c}\right)$. Consequently, if we are to predict a rating value for a requesting user $u_{r}$ about a target item $i t_{t}$, we compute the average of the rating values contained in $G_{r}$, each weighted with the respective edge weights in $G_{s}$. When computing this weighted average, we only need the continuous proximity values for the rating user to all other users in the local graph. The similarity between other users does not affect the aggregation and thus no continuous proximity information needs to be passed on when ratings are exchanged.

\section{Equivalence to Existing Algorithms}

As explained in the previous section, users of our recommender system exchange tuples when they are in spatio-temporal proximity. Each user maintains a graph $G_{\text {local }}$ where the nodes in $U$ represent users previously met and the nodes in $I$ represent all items rated by these users or the local user. In this section we first explain why such a local graph is sufficient to perform user-based collaborative filtering. Secondly, we show that the resulting algorithm resolves scalability issues for which user-based approaches have frequently been criticised.

We first look at a simple form of traditional user-based CF where rating values are set to 1 if a user has consumed an item and 0 otherwise. For example, the Amazon online store interprets the purchase of an item as an expression of a binary opinion about it. Thus, each user is represented by a binary vector containing entries for all items. A server maintains the set of user vectors based on which ratings are predicted. The similarity between two users is computed as the number of vector entries both have set to 1 . The prediction is the result of aggregating the ratings of all users about the target item, each weighted with the similarity between the requesting and rating user. Consequently, the prediction is based on the set of users that have consumed at least one item which the requesting user has also consumed. All other users are not included because their ratings are weighted with a zero-valued similarity.

A user vector is a set of rating tuples where the user entry contains the represented user. The tuples stored on the server define a graph $G_{\text {global }}$ which, in contrast to a local graph, includes all participating users and items consumed by any user. Therefore, a local graph is a subgraph of the global graph while the global graph is a union of all local graphs. In fact, a local graph belonging to a particular user $u_{i}$ can be extracted from the global graph as follows. We use superscript notations $g$ and $l$ to indicate that a node or edge set belongs to the global or local graph, respectively. An edge is denoted as $(p, q)$ where $p$ and $q$ are the adjacent nodes. Finally, $w_{(p, q)}$ refers to the weight of an edge $(p, q)$.

$$
\begin{aligned}
U^{l} & =\left\{u \mid u \in U^{g} \wedge\left(u_{i}, u\right) \in E_{s}^{g} \wedge w_{\left(u_{i}, u\right)}>0\right\} \cup u_{i} \\
I^{l} & =\left\{i \mid i \in I^{g} \wedge(u, i) \in E_{r}^{g} \wedge u \in U^{l}\right\} \\
E_{r}^{l} & =\left\{(u, i) \mid(u, i) \in E_{r}^{g} \wedge u \in U^{l} \wedge i \in I^{l}\right\} \\
E_{s}^{l} & =\left\{\left(u_{i}, u\right) \mid\left(u_{i}, u\right) \in E_{s}^{g}\right\}
\end{aligned}
$$


Equation 1 states that we take all users in $U^{g}$ which are connected to $u_{i}$ by a similarity edge with a weight greater than zero. Equation 2 selects all items that are connected to a user selected in Eq. 1. Equation 3 selects all rating edges whose adjacent user and item have been selected by the previous two equations. Finally, Eq. 4 accounts for the fact that similarity edges are not exchanged. Thus, only similarity edges between $u_{i}$ and the other users are selected. In Fig. 3, we highlight the local graph as part of the global graph. Nodes and edges not belonging to the local graph are drawn with a dashed line.

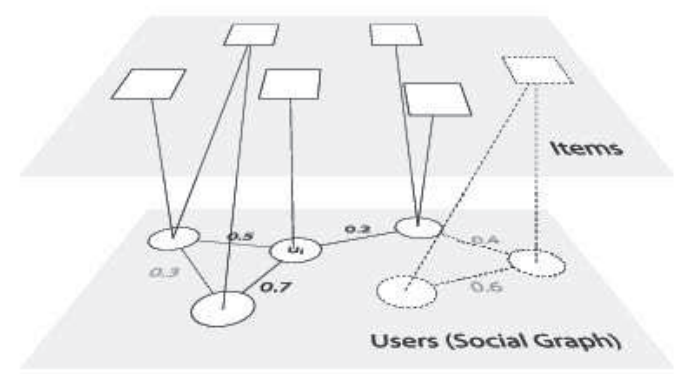

Fig. 3. Local graph as a subgraph of the global graph

Simple traditional $\mathrm{CF}$ outlined above predicts a rating for a requesting user $u_{r}$ about a target item $i_{t}$ as

$$
\frac{1}{\left|\left(u, i_{t}\right) \in E_{r}^{g}:\left(u_{r}, u\right) \in E_{s}^{g}\right|} \sum_{\left(u, i_{t}\right) \in E_{r}^{g}} w_{\left(u, i_{t}\right)} \cdot w_{\left(u_{r}, u\right)}
$$

where the aggregation is a weighted average of the ratings. Now we want to show that all rating and social edges included in the aggregation also exist in the local graph belonging to $u_{r}$. The underlying intuition is that users from whom ratings are aggregated have in common the fact that they consumed items also consumed by the requesting user. Hence, if users exchange their own ratings whenever they consume the same item, the set of users from whom opinions are collected and thus are available in the local graph is equivalent to the set of users selected in the global graph by traditional user-based CF. In order to prove this equivalence, we have to show that all rating and social edges included in the sum in Eq. 5 also exist in the local graph. This is obvious for the social edges because all edges $w_{\left(u_{r}, u\right)} \in E_{s}^{g}$ have been selected by Eq. 4 and, since we are considering the local graph belonging to $u_{r}$, it holds that $u_{i}=u_{r}$. In order to simplify this proof of equivalence, we can now leave out the weighting of each rating. Therefore we rewrite Eq. 5 as

$$
\frac{1}{\left|\left(u, i_{t}\right) \in E_{r}^{g}:\left(u_{r}, u\right) \in E_{s}^{g}\right|} \sum_{\left(u, i_{t}\right) \in E_{r}:\left(u_{r}, u\right) \in E_{g}^{g} \wedge w_{\left(u_{r}, u\right)}>0} w_{\left(u, i_{t}\right)}
$$


where the condition of the sum ensures rating edges are included only from rating users that have a non-zero similarity to the requesting user. Now it is apparent that the rating edges included in the aggregation are also contained in the local graph since $E_{r}^{l}$ is extracted from $E_{r}^{g}$ by applying Eq. 1, 2 and 3 consecutively, while the selection criteria of the sum is equivalent to Eq. 1.

Since a local graph contains all edges used by traditional user-based CF for rating predictions based on the global graph, the same results can be obtained from the local graph alone thereby eliminating the need for a central server to store all user vectors to compute similarities between users which is considered the main bottleneck in traditional $\mathrm{CF}$.

\section{Conclusions}

We have presented a technique for user-based collaborative filtering that exploits an opportunistic mode of information sharing resulting from ad-hoc peer-to-peer networking. Only users in spatio-temporal proximity are able to exchange ratings and we have shown how this provides a natural filtering based on social contexts. The resulting selection of similar users renders the computation of similarities and selection of most similar users unnecessary which resolves sparsity and scalability issues frequently associated with user-based collaborative filtering.

\section{References}

1. Lawrence, J., Payne, T.R., Roure, D.D.: Co-presence Communities: Using Pervasive Computing to Support Weak Social Networks. In: Proc. Intl. Workshop on Distributed and Mobile Collaboration (2006)

2. Counts, S., Geraci, J.: Incorporating Physical Co-presence at Events into Digital Social Networking. In: Extended Abstracts on Human Factors in Computing Systems (2005)

3. Resnick, P., Iacovou, N., Suchak, M., Bergstrom, P., Riedl, J.: GroupLens: An Open Architecture for Collaborative Filtering of Netnews. In: Proc. Conf. on Computer Supported Cooperative Work (1994)

4. Shardanand, U., Maes, P.: Social Information Filtering: Algorithms for Automating "Word of Mouth". In: Proc. Intl. Conf. on Human Factors in Computing Systems (1995)

5. Hill, W., Stead, L., Rosenstein, M., Furnas, G.: Recommending and Evaluating Choices in a Virtual Community of Use. In: Proc. Intl. Conf. on Human Factors in Computing Systems (1995)

6. Terveen, L.G., Hill, W.C., Amento, B., McDonald, D., Creter, J.: Building TaskSpecific Interfaces to High Volume Conversational Data. In: Proc. Conf. on Human Factors in Computing Systems (1997)

7. Sarwar, B., Karypis, G., Konstan, J., Reidl, J.: Item-based Collaborative Filtering Recommendation Algorithms. In: Proc. Intl. Conf. on World Wide Web (2001)

8. Wang, J., Pouwelse, J., Lagendijk, R.L., Reinders, M.J.T.: Distributed Collaborative Filtering for Peer-to-Peer File Sharing Systems. In: Proc. Symp. on Applied Computing 
9. Miller, B.N., Konstan, J.A., Riedl, J.: PocketLens: Toward a Personal Recommender System. ACM Trans. Inf. Syst. 22(3), 437-476 (2004)

10. Tveit, A.: Peer-to-peer Based Recommendations for Mobile Commerce. In: Proc. Intl. Workshop on Mobile Commerce

11. Mirza, B.J., Keller, B.J., Ramakrishnan, N.: Studying Recommendation Algorithms by Graph Analysis. J. Intell. Inf. Syst. 20(2), 131-160 (2003) 